Revista de Matemática: Teoría y Aplicaciones 1998 5(1) : 65-71

CIMPA - UCR - CCSS ISSN: 1409-2433

\title{
MÉTODOS NO ESTÁNDAR EN EL PROBLEMA DE LA PARADA ÓPTIMA
}

\author{
JAIME LOBO*
}

Recibido: 21 Diciembre 1997

\begin{abstract}
Resumen
Se analiza el problema de la parada óptima por medio de las técnicas del análisis no standar. Gracias al concepto no estándar de tiempo casi-óptimo se logra deducir la existencia de tiempos óptimos para los problemas de horizonte finito e infinito. El enfoque adoptado para el análisis no estándar es el de Internal Set Theory.
\end{abstract}

Palabras-clave: problema de parada óptima, horizontes finito e infinito, Internal Set Theory, tiempo casi-óptimo, tiempo óptimo, integrabilidad según Loeb-Nelson.

\begin{abstract}
We analize the optimal-stopping problem by the use of non standard analysis techniques. Using the non standard concept of quasi-optimal time, we prove the existence of optimal times for the problems of finite and infinite horizon. The approach used in non standard analysis is that of the Internal Set Theory.
\end{abstract}

Keywords: optimal-stopping problem, finite and infinite horizon, Internal Set Theory, quasi-optimal time, optimal time, Loeb-Nelson integrability.

AMS Subject Classification: 60G40, 62L15, 26E35, 03H05

\section{Introducción}

La teoría de la parada óptima consiste en el problema siguiente: se considera en un espacio de probabilidades $(\Omega, A, P)$ una sucesión de variables integrables $Z=\left(Z_{n}\right)_{n \in \mathbb{N}}$ adaptada a una filtración $F=\left(F_{n}\right)_{n \in \mathbb{N}}: F_{n+1} \supset F_{n}$. Sea $\mathcal{T}$ el conjunto de tiempos de parada de $F$ casi siempre finitos:

$$
\mathcal{T}=\left\{t: \Omega \rightarrow \mathbb{N}, t \text { es c.s. finito y }\{t=n\} \text { es evento de } F_{n} \text { para todo } n \in \mathbb{N}\right\}
$$

\footnotetext{
*Escuela de Matemática, Universidad de Costa Rica, 2060 San José, Costa Rica.
} 
Si $C$ es un subconjunto de $\mathcal{T}$ tal que para toda $t$ en $C$ la variable $Z_{t}$ es integrable, se trata de estudiar el valor de $\sup \left\{E\left(Z_{t}\right), t \in C\right\}$ y eventualmente encontrar un tiempo en $C$ donde se alcance este valor. Llamaremos un tal tiempo de paro tiempo óptimo del problema de parada óptima definido por el conjunto $C$. Las condiciones de integrabilidad sobre $Z$ permiten abordar siempre el problema de horizonte finito, de horizonte $N$, que se define tomando $C=\mathcal{T}^{N}=$ tiempos de parada de $F$ menores que $N$. Bajo condiciones más fuertes en la sección 3 se abordará el problema de horizonte infinito en donde se toma $C=\mathcal{T}$.

La teoría clásica de la parada óptima, expuesta por ejemplo en [4] o [7], trata este problema introduciendo el concepto de supremo esencial de una familia de variables aleatorias. Esto es necesario porque en general no es posible definir una variable aleatoria como $\sup _{t \in C} Z_{t}$, ya que el conjunto de índices $C$ es en general no numerable. En este trabajo se dará una nueva presentación del problema de la parada óptima recurriendo a ciertos resultados derivados del análisis no estándar que evita ciertas dificultades técnicas de la teoría clásica, entre ellas los del supremo esencial antes mencionado. Seguimos el enfoque sintáctico de la Internal Set Theory (IST), que permite aplicar los razonamientos no estándar a los objetos de estudio (espacios de probabilidad, procesos, etc.) sin necesidad de las extensiones que usualmente se requieren en los enfoques no sintácticos.

Los resultados más importantes de la IST necesarios para nuestros propósitos serán recordados en la sección 2 donde se introduce el concepto nuevo de tiempo casi-óptimo. En las secciones siguientes se establece la existencia (para el problema de horizonte finito primeramente y luego para el horizonte infinito) de tiempos casi-óptimos a partir de los cuales se derivan las soluciones clásicas.

\section{Preliminares sobre la IST y la parada óptima}

Al adoptar el concepto de conjunto estándar tal como es definido en la IST, teoría desarrollada en [1] o [3], es pertinente recordar que gracias al axioma del transfer y otros axiomas de la IST muchas propiedades clásicas admiten una caracterización no estándar cuando son aplicadas a dichos objetos. Gracias al transfer podremos suponer que las variables que intervienen en la formulación de un teorema dado son estándar, por lo que supondremos en varias ocasiones que se cumple la condición $(S)$ siguiente:

$(S):(\Omega, A, P), Z$, y la filtración $F$ son estándar

Además de la IST echaremos mano del siguiente corolario del axioma de idealización (ver [1]) que llamaremos:

IST1: Para todo conjunto $E$ existe un conjunto finito $F$ que contiene a todos los elementos estándar de $E$.

Llamaremos tiempo casi-óptimo del problema de parada óptima definido por $C$ a un tiempo de parada $\tau$ de $C$ tal que $E\left(Z_{t}\right) \approx$ máx $_{t \in C} E\left(Z_{t}\right)$.

También serán de utilidad algunos resultados no estándar sobre integración. Una variable aletoria $X$ es Loeb-Nelson integrable si su esperanza es limitada y para cualquier even- 
to $A$ de probabilidad infinitesimal (se dice evento raro) se tiene $E\left(|X| 1_{A}\right) \approx 0$. Aceptaremos además los teoremas siguientes (ver [5], [1]):

IST2: Una variable aleatoria estándar integrable definida en un espacio estándar es Loeb-Nelson integrable

IST3: Si $X, Y$ son variables aleatorias, $|X| \leq|Y|$ y $Y$ es Loeb-Nelson integrable entonces $X$ lo es también

IST4: Si $X, Y$ son variables aleatorias Loeb-Nelsos integrables y $X \approx Y$ casi siempre entonces $E(X) \approx E(Y)$

De la teoría de la parada óptima recurriremos a un lema técnico (ver [4], [7]), que se enuncia así:

OPT1: sea $\left(A_{n}\right)_{n \leq \mathbb{N}}$ una suseción que cumple:

$$
A_{N}=Z_{N}, A_{n}=\operatorname{máx}\left(Z_{n}, E^{n}\left(A_{n}\right)\right),\left(\text { resp. } A_{n} \leq \operatorname{máx}\left(Z_{n}, E^{n}\left(A_{n}\right)\right)\right) .
$$

Entonces si $\tau$ es el tiempo de parada definido por

$$
\tau=\min \left\{n: n \leq N, A_{n}=Z_{n}\right\}
$$

el proceso $B_{n}=A_{\operatorname{mín}(n, \tau)}$ es una martingala de $F$ (resp. submartingala de $F)$.

\section{Tiempos casi-óptimos y óptimos del problema de hori- zonte finito}

En virtud del IST1 existe un conjunto finito $\wp^{N}$ en $\tau^{N}$ que contiene a todos los tiempos de parada estándar de $\tau^{N}$. El máximo de una familia de variables indexadas por submartingala de $F$. Gracias a esto se obtiene:

Teorema 1 Bajo las condiciones $(S)$ y siendo $N$ es estándar:

$$
E\left(Z_{v^{N}}\right) \geq \operatorname{máx}_{t \in \wp^{N}} E\left(Z_{t}\right)
$$

y por lo tanto $E\left(Z_{v^{N}}\right) \approx \operatorname{máx}_{t \in \tau^{N}} E\left(Z_{t}\right)$. El tiempo $v^{N}$ es pues casi-óptimo del problema de horizonte $N$.

Prueba: La última relación es consecuencia inmediata del primer resultado, del lema 1 y del hecho de que $v^{N}$ es elemento de $\tau^{N}$. Para la primera notamos primero que por definición se tiene $Z_{v^{N}}=V^{N}\left(v^{N}\right)$. Por otro lado $E\left(V^{N}\left(v^{N}\right)\right)=E\left(Y_{N}\right) \geq E\left(Y_{1}\right)$, donde la desigualdad se da por el hecho de ser $\left(Y_{n}\right)$ una submartingala. Por otro lado $E\left(Y_{1}\right)=$ $E\left(\right.$ máx $\left._{t \in \wp^{N}} E^{1}\left(Z_{t}\right)\right) \geq \operatorname{máx}_{t \in \wp^{N}} E\left(Z_{t}\right)$, y se concluye.

Si el tiempo $v^{N}$ fuera además estándar sería óptimo, pues bajo las condiciones $(S)$ tanto el valor de $E\left(Z_{v^{N}}\right)$ como el de máx $\operatorname{me\tau }^{N} E\left(Z_{t}\right)$ son estándar y siendo equivalentes deben 
ser iguales (ver[1]). Sin embargo no podemos asegurar que en general $v^{N}$ sea estándar, pues en su definición interviene el conjunto $\wp^{N}$ que no es necesariamente estándar. Podemos sin embargo encontrar un tiempo óptimo de las desigualdades obtenidas en el lema 2 . Introducimos una nueva sucesión $W^{N}(n), n=1, \ldots, N$ definida de esta manera (recurrencia hacia atrás de Bellman, ver [4]):

$$
W^{N}(N)=Z_{N}, n<N: W^{N}(n)=\operatorname{máx}\left(Z_{n}, E^{n}\left(W^{N}(n+1)\right)\right)
$$

Esta sucesión de variables es estándar bajo las condiciones $(S)$ y para $N$ estándar. Se sigue que en este caso el tiempo de parada $\mu^{N}$ definido por:

$$
\mu^{N}=\inf \left\{n: n \leq N: W^{N}(n)=Z_{n}\right\}
$$

es estándar también.

Establecemos ahora que $E\left(Z_{v^{N}}\right)=E\left(Z_{\mu^{N}}\right)$. La desigualdad $E\left(Z_{v^{N}}\right) \geq E\left(Z_{\mu^{N}}\right)$ proviene del teorema 1. Para obtener el otro sentido observemos primero que por definición se tiene:

$$
E\left(Z_{\mu^{N}}\right)=E\left(W^{N}\left(\mu^{N}\right)\right)
$$

De OPT1 la sucesión $Y \sim_{n}=W^{N}\left(\operatorname{mín}\left(n, \mu^{N}\right)\right)$ es una martingala, lo que implica:

$$
E\left(W^{N}\left(\mu^{N}\right)\right)=E\left(Y \sim_{\mu^{N}}\right)=E\left(Y \sim_{v^{N}}\right)
$$

Por otro lado no es difícil mostrar que para $n \leq N: W^{N}(n) \geq V^{N}(n)$, y por ende $\mu^{N} \geq v^{N}$ y en consecuencia:

$$
E\left(Y \sim_{v^{N}}\right)=E\left(W^{N}\left(v^{N}\right)\right) \geq E\left(V^{N}\left(v^{N}\right)\right)=E\left(Z_{v^{N}}\right)
$$

Las relaciones (1), (2), (3) implican entonces que $E\left(Z_{v^{N}}\right) \leq E\left(Z_{\mu^{N}}\right)$. Se tiene finalmente $E\left(Z_{v^{N}}\right)=E\left(Z_{\mu^{N}}\right)$.

De esta última igualdad, del teorema 1 y los comentarios que le siguen se deduce $\wp^{N}$ es una variable aleatoria, y en particular para cualquier subtribu $\mathcal{A}$ de $A$ existe la variable aleatoria: $\operatorname{máx}_{t \in \wp^{N}} E\left(Z_{t} \mid \mathcal{A}\right)$. Podemos suponer además que $\wp^{N}$ es estable por la operación sup: para $t, t^{\prime}$ en $\wp^{N}, \sup \left(t, t^{\prime}\right) \in \wp^{N}$. En efecto si $\wp^{N}$ es cualquier conjunto finito conteniendo los tiempos de parada estándar de $\tau^{N}$ basta considerar el menor conjunto estable de $\tau^{N}$ bajo la operación sup que contenga $\wp^{N}$, que sigue siendo finito.

No es difícil establecer por el principio del transfer y la caracterización del extremo superior de un conjunto de reales estándar (ver [3]) lo siguiente:

Lema 1 Bajo las condiciones $(S)$ se tiene:

$$
\operatorname{máx}_{t \in \wp^{N}} E\left(Z_{t}\right) \approx \operatorname{máx}_{t \in \tau^{N}} E\left(Z_{t}\right)
$$

En el lema 1, el miembro izquierdo es un máximo sobre un conjunto finito, el conjunto $\wp^{N}$, y por lo tanto se alcanza en un tiempo de parada $\tau$, de manera tal que:

$$
E\left(Z_{t}\right) \approx \operatorname{máx}_{t \in \tau^{N}} E\left(Z_{t}\right)
$$


Un tal tiempo $\tau$ es entonces casi-óptimo para el problema de parada óptima de horizonte $N$. Es de observar que sin las condiciones $(S)$ no es posible obtener este resultado con la ayuda única del transfer, y por ende asegurar un casi-óptimo del problema. Sin embargo el mismo principio permitirá establecer y aun construir tiempos óptimos como se probará a continuación.

Describimos ahora un tiempo de parada casi-óptimo. Para esto consideremos las variables aleatorias $V^{N}(n), n=1, \ldots, N$ definidas por:

$$
V^{N}(N)=Z_{N}, V^{N}(n)=\operatorname{máx}_{t \in \wp^{N}, t \geq n} E^{n}\left(Z_{t}\right)
$$

donde $E^{n}\left(\right.$ ) denota la esperanza condicional con respecto a $F_{n}$. Se tiene entonces:

Lema 2 Si $N$ es estándar, para $n \leq N$ se cumple: $V^{N}(n) \leq \operatorname{máx}\left(Z_{n}, E^{n}\left(V^{N}(n+1)\right)\right.$

Prueba: Sea $t$ en $\wp^{N}, t \geq n$. Entonces $Z_{t}=Z_{t} 1_{\{t=n\}}+Z_{t} 1_{\{t>n\}}$. En $\{t>n\}$ se puede escribir $t=\sup (t, n+1)=t^{\prime}$, que es elemento de $\wp^{N}$ con $t^{\prime} \geq n+1$, pues $n+1$ es estándar y que $\wp^{N}$ es estable por la operación sup. Si tomamos la esperanza $E^{n}$ ( ) en esta igualdad:

$$
E^{n}\left(Z_{t}\right)=E^{n}\left(Z_{n}\right) 1_{\{t=n\}}+E^{n}\left(Z_{t}\right) 1_{\{t>n\}}=Z_{n} 1_{\{t=n\}}+E^{n}\left(Z_{t}\right) 1_{\{t>n\}}
$$

y como $E^{n}\left(Z_{t}\right)=E^{n}\left(E^{n+1}\left(Z_{t}\right)\right) \leq E^{n}\left(V^{N}(n+1)\right)$, por definición de la variable $V^{N}(n+1)$, se obtiene

$$
E^{n}\left(Z_{t}\right) \leq Z_{n} 1_{\{t=n\}}+E^{n}\left(V^{N}(n+1)\right) 1_{\{t>n\}} \leq \operatorname{máx}\left(Z_{n}, E^{n}\left(V^{N}(n+1)\right) .\right.
$$

Definimos ahora un tiempo de parada $v^{N}$ por:

$$
v^{N}=\inf \left\{n: n \leq N: V^{N}(n)=Z_{n}\right\}
$$

Del lema 2 y de OPT1 se deduce que el proceso $Y_{n}=V^{N}\left(\operatorname{mín}\left(n, v^{N}\right)\right)$ es una que bajo las condiciones $(S)$, y $N$ siendo entero estándar, el tiempo de parada $\mu^{N}$ es óptimo: $E\left(Z_{\mu^{N}}\right)=$ $\operatorname{máx}_{t \in \tau^{N}} E\left(Z_{t}\right)$. Ahora bien siendo esta propiedad de optimalidad una propiedad estándar, del resultado obtenido bajo condiciones $(S)$ y del transfer se deduce:

Teorema 2 Para todo $N$ entero el tiempo de parada $\mu^{N}$ es óptimo:

$$
E\left(Z_{\mu^{N}}\right)=\operatorname{máx}_{t \in \tau^{N}} E\left(Z_{t}\right) \text {. }
$$

\section{Tiempos casi-óptimos y óptimos del problema de hori- zonte infinito}

Abordemos ahora el problema de parada óptima de horizonte infinito, es decir el estudio de $S_{\infty}=\sup \left\{E\left(Z_{t}\right), t \in \tau\right\}$. Para garantizar que $S_{\infty}$ está bien definido y es finito supondremos la condición

$$
H: \sup _{n \in \mathbb{N}}\left|Z_{n}\right| \text { es integrable }
$$

(bajo estas condiciones el problema se estudia en [6]).

Buscamos la existencia de posibles tiempos casi-óptimos y óptimos en el sentido clásico. Para esto supondremos primeramente que se cumplen las condiciones $(S)$. 
Lema 3 Bajo las condiciones $(S)$ toda variable de la forma $Z_{\tau}$, donde $\tau$ pertenece a $\mathcal{T}$, es Loeb-Nelson integrable.

Prueba: La variable $\left|Z_{\gamma}\right|$ es acotada por la variable $X=\sup _{n \in \mathbb{N}}\left|Z_{n}\right|$, que por la condición $H$ y la hipótesis del lema es integrable y estándar. Pero entonces $X$ es Loeb-Nelson integrable por IST2 y luego por IST3 $\left|Z_{\gamma}\right|$ lo es también.

Teorema 3 Bajo las condiciones $(S)$ y para $N \approx \infty, S_{\infty} \approx S_{N}$. En particular $\mu^{N}$ es tiempo casi-óptimo del problema de horizonte infinito.

Prueba: Bajo $(S)$ se tiene que $S_{\infty}$ es estándar y por transfer se obtiene que para cada $\epsilon$ estándar $>0$ existe $\gamma$ estándar en $\tau$ tal que $\left|S_{\infty}-E\left(Z_{\gamma}\right)\right|<\epsilon$. Ahora bien, siendo $\gamma$ estándar casi siempre finito se cumple que $P(\gamma \geq N)$ es infinitesimal cada vez que $N$ es ilimitado. Por otro lado por el lema 3 las variables $Z_{\gamma}$ y $Z_{\min (\gamma, N)}$ son Loeb-Nelson integrables y por lo tanto $E\left(Z_{\gamma} 1_{\{\gamma \geq N\}} \approx 0, E\left(Z_{\min (\gamma, N)} 1_{\{\gamma \geq N\}}\right) \approx 0\right.$. Se deduce entonces que $E\left(Z_{\gamma}\right)=E\left(Z_{\gamma} 1_{\{\gamma \geq N\}}\right)+E\left(Z_{\gamma} 1_{\{\gamma<N\}}\right) \approx E\left(Z_{\gamma} 1_{\{\gamma<N\}}\right) \approx E\left(Z_{\min (\gamma, N)}\right)$ es decir $E\left(Z_{\gamma}\right) \approx E\left(Z_{\operatorname{mín}(\gamma, N)}\right)$. Pero $E\left(Z_{\min (\gamma, N)}\right) \leq S_{N}$, pues el tiempo $\operatorname{mín}(\gamma, N)$ pertenece a $\tau^{N}$. De la escogencia del tiempo $\tau$ se obtiene entonces $\left|S_{\infty}-E\left(Z_{\gamma}\right)\right|<\epsilon$. Pero $\epsilon$ siendo estándar positivo arbitrario, la desigualdad anterior implica que $S_{\infty} \approx S_{N}$, de donde concluimos. El último aserto es consecuencia de lo anterior y del teorema 2 .

Reinterpretamos el resultado anterior en términos clásicos: la sucesión $\left(S_{n}\right)$ siendo estándar, la propiedad $S_{\infty} \approx S_{N}$ para todo $N$ ilimitado significa que $\left(S_{n}\right)$ converge al valor de $S_{\infty}$ (caracterización no estándar de convergencia de sucesiones). Aplicando transfer deducimos el resultado clásico ([4], [7]):

Teorema 3' La sucesión $\left(S_{n}\right)$ converge al valor $S_{\infty}$. En particular para cada $\epsilon>0$ existe un entero $N_{\epsilon}$ tal que $\mu N_{\epsilon}$ es $\epsilon-$ solución del problema:

$$
\left|S_{\infty}-E\left(Z_{\mu N_{\epsilon}}\right)\right|<\epsilon
$$

Los teoremas 3 y 3' permiten solamente caracterizar los tiempos casi-óptimos del problema de horizonte infinito como tiempos óptimos de horizonte finito. Para asegurar realmente la existencia de tiempos óptimos consideremos el tiempo $\mu^{\infty}=\operatorname{lím}_{n \rightarrow \infty} \mu^{n}$, donde el límite tiene sentido dado que $\left(\mu^{n}\right)_{n}$ es una sucesión creciente de variables aleatorias. El tiempo $\mu^{\infty}$ no es necesariamente casi infinito aún bajo las condiciones $H$ (ver[4]). Sin embargo si así fuera se cumple:

Teorema 4 Si el tiempo $\mu^{\infty}$ es casi siempre finito, entonces $\mu^{\infty}$ es tiempo óptimo del problema de horizonte infinito.

Prueba: Por transfer basta suponer que se cumplen las condiciones $(S)$. Puesto que $\mu^{\infty}$ es casi siempre finito la variable $Z_{\mu^{\infty}}$ está bien definida y es estándar. Siendo este tiempo el límite de la sucesión estándar $\left(\mu^{n}\right)_{n}$, de valores enteros, la caracterización no estándar de convergencia en probabilidad implica que $\mu^{N}=\mu^{\infty}$ para todo $N \approx \infty$ fuera de un 
evento raro. En consecuencia $Z_{\mu^{\infty}}=Z_{\mu^{N}}$ fuera de este evento raro, y siendo ambas LoebNelson integrables (lema 3) aplicando IST4 obtenemos $E\left(Z_{\mu^{\infty}}\right) \approx E\left(Z_{\mu^{N}}\right)$. Del teorema 3 se deduce que $E\left(Z_{\mu^{\infty}}\right) \approx S_{\infty}$, y como en esta relación ambas cantidades son estándar deben ser entonces iguales y se concluye.

El estudio precedente del problema de la parada óptima de horizonte infinito se ha presentado bajo el supuesto que se cumplen las condiciones $H$ y sería interesante extenderlo a condiciones más generales, tales como las dadas en [4], conservando las ideas del análisis no estándar. Por otra parte queda planteado el problema de tratar la teoría de la parada óptima en tiempo continuo con el enfoque no estándar.

\section{Referencias}

[1] E. Nelson (1977) "Internal Set Theory: A New Approach to Nonstandard Analysis", Bulletin of the American Mathematical Society 83(6).

[2] E. Nelson (1987) Radically Elementary Probability Theory. Princeton.

[3] Diener, F.; Reeb, G. (1989) Analyse Non Standard. Hermann, Paris.

[4] Chow, Robbins, Slegmund (1971) Great Expectations: The Teory of Optimal Stopping. Houghton.

[5] Cartle, P.; Feneyrol-Perrin (1988) "Comparaison des Diverses Théories D'intégration en Analyse non Standard", Comptes Rendus de L'Académie des Sciences de Paris, Tomo 307, Serie I.

[6] Dacunha-Castelle, D.; Duflo, M. (1983) Probabilités et Statistiques, 2. Problèmes à Temps Mobile. Masson, Paris.

[7] Neveu, J. (1972) Martingales à Temps Discret. Masson, Paris. 